\title{
Consciência fonológica e linguagem escrita: efeitos de um programa de intervenção
}

\section{Phonological awareness and written language: effects of an intervention program}

\author{
Maria José dos Santos ${ }^{1}$ \\ Maria Regina Maluf ${ }^{2}$
}

\begin{abstract}
RESUMO
A consciência fonológica, entendida como habilidade de reflexão e manipulação dos sons da fala, está relacionada ao êxito na aquisição da linguagem escrita. Esta pesquisa teve como objetivos avaliar a eficácia de um programa de intervenção para desenvolver habilidades metafonológicas e facilitar a aprendizagem da escrita em crianças falantes do português do Brasil. Participaram 90 crianças de 5 a 6 anos que frequentavam classes de alfabetização. Foi utilizado delineamento quase experimental em três fases: pré-teste, intervenção e pós-teste, com aplicação de provas às crianças. Os resultados mostraram que as habilidades metafonológicas têm papel facilitador no início do processo de aquisição da linguagem escrita e podem ser desenvolvidas por meio de programas de intervenção em diferentes condições de aplicação.

Palavras-chave: onsciência fonológica; alfabetização; programa de intervenção.
\end{abstract}

\begin{abstract}
The phonological awareness, understood as the ability of reflection and manipulation of speech sounds, is related to succeeding in the written language acquisition process. This research aimed at evaluating the effectiveness of an intervention program to develop metaphonological skills and aid the learning of writing for Brazilian Portuguese-speaking children. A total of
\end{abstract}

1 Doutora em Psicologia da Educação. Professora da Universidade Federal de Goiás no Campus Catalão - Brasil.. E-mail: majossantos@hotmail.com.

2 Doutora pela Universite Catholique de Louvain, Bélgica. Professora titular da Pontifícia Universidade Católica de São Paulo - Brasil: Programa de Pós-Graduação em Psicologia da Educação. 
90 children between 5 and 6 years old who attended the literacy classes participated in this research. A quasi-experimental design was used in three steps: pre-test, intervention and post-test, with the application of exams to the children. The results showed that the metaphonological skills have an aiding role at the beginning of the written language acquisition process, and that they can be developed through intervention programs in different application conditions.

Keywords: phonological awareness; literacy; intervention program.

Uma das importantes contribuições da pesquisa sobre aquisição da linguagem escrita foi a descoberta da relação entre habilidades de reflexão sobre a linguagem, designadas como metafonológicas, e a aprendizagem da linguagem escrita (BRADLEY; BRYANT, 1983; CARDOSO-MARTINS, 1995; HESTER; HODSON, 2004; MORAIS; CARY; ALEGRIA; BERTELSON, 1979; MUTER; HULME; SNOWLING, 1998; NATION; HULME, 1997; SNOWLING; HULME; SMITH; THOMAS, 1994).

As habilidades metafonológicas dizem respeito à identificação e produção de rima e aliteração, segmentação de frases em palavras (segmentação lexical), segmentação de palavras em sílabas e de palavras em fonemas (segmentação fonêmica). Essas habilidades diferem quanto ao nível de exigência cognitiva (STANOVICH, 1992). Assim, por exemplo, já foi demonstrado que a identificação e produção de rimas e aliterações exigem sensibilidade à similaridade da sequência fonológica e requerem pouca capacidade analítica (CARDOSOMARTINS, 1995; MUTER; HULME; SNOWLING; TAYLOR, 1998; STAHL; MURRAY, 1994), enquanto que a segmentação de palavras em fonemas exige representação explícita dos segmentos fonêmicos (ANTHONY; LONIGAN; DRISCOLL et al., 2003; HATCHER; HULME, 1999; MURRAY; 1998; TORGESEN; DAVIS, 1996).

A identificação de rimas e aliterações, assim como a segmentação de unidades silábicas, podem se desenvolver espontaneamente, independentemente do ambiente escolar (LIBERMAN; SHANKWEILLER; FISCHER; CARTER, 1974; TREIMAN, 1992). Porém, habilidades mais complexas como, por exemplo, a síntese ou segmentação fonêmica, parecem depender da aprendizagem da leitura e escrita (MORAIS, 1996; PERFETTI; BECK; BELL; HUGUES, 1987).

As evidências da importância dessas habilidades metafonológicas para a aquisição da linguagem escrita vêm de diferentes tipos de pesquisas. Com frequência são encontrados, na literatura da área, estudos de tipo correlacional (LIBERMAN et al., 1974; MANN; LIBERMAN, 1984; JUEL, 1988). Estudos de tipo longitudinal, que investigam relações de precedência entre habili- 
dades metafonológicas e de leitura e escrita (JONG; VAN DER LEIJ, 1999; WAGNER; TORGESEN, 1987; WESSELING; REITSMA, 2001; PERFETTI et al., 1987), são menos frequentes. Pesquisas que incorporam programas de intervenção em habilidades metafonológicas demonstram as influências desta habilidade no processo de aquisição da linguagem escrita (BALL; BLACHMAN, 1991; BRADLEY; BRYANT, 1983; LUNDBERG; FROST; PETERSEN, 1988; TORGESEN; DAVIS, 1996; TORGESEN; MORGAN; DAVIS, 1992; TORGESEN; WAGNER; RASHOTTE et al., 1999).

Estudos de intervenção são importantes para avançar na compreensão das relações entre habilidades metafonológicas e aquisição da linguagem escrita. Embora sejam relativamente frequentes na literatura estrangeira da área, são ainda pouco numerosos com falantes do português do Brasil (CAPELLINI; CIASCA, 2000; CAPOVILLA; CAPOVILLA, 2000; SANTOS, 2004; CARNIO; SANTOS, 2005; PAULA; MOTA; KESKE-SOARES, 2005).

Programas de intervenção, que combinam atividades para o desenvolvimento de habilidades metafonológicas, e atividades que visam à aquisição do valor sonoro e nome das letras, parecem ter efeito superior na aquisição da leitura e escrita, quando comparados a programas com atividades desses componentes desenvolvidos separadamente (SCHNEIDER; KÜSPERT; ROTH et al., 1997). A utilização de letras como apoio em atividades de segmentação de palavras favorece a consciência fonológica (HOHN; EHRI, 1983; PAULA; MOTA; KESKE-SOARES, 2005). Experimentos mostraram que as crianças que receberam o apoio de letras, durante a realização de atividades de segmentação de palavras, beneficiaram-se mais do que as que não receberam esse apoio. Os mesmos autores mostram que a utilização de letras, durante atividades de intervenção em consciência fonêmica, produz melhor compreensão da natureza da tarefa e fornece pistas de como desenvolvê-la, favorecendo assim a distinção da unidade sonora a ser segmentada.

Resultados obtidos por Cunningham (1990) evidenciaram que a aplicação de um programa de intervenção em habilidades metafonológicas, com instrução explícita de como e onde utilizar essas habilidades, teve influência sobre o desempenho em leitura e escrita apenas quando articulado com programas escolares de leitura. Neste caso, as crianças parecem ter mais oportunidades de utilizar e aplicar as habilidades aprendidas durante a implementação do programa.

Diferentes habilidades metafonológicas produzem efeitos diversos na aprendizagem da linguagem escrita. Torgesen, Morgan e Davis (1992) compararam os efeitos da intervenção em habilidades de análise e de síntese de fonemas. Os resultados sugeriram que o treinamento em ambas as habilidades, de análise e de síntese, produz bons resultados na segmentação de palavras em fonemas. Em contraste, o treinamento apenas em habilidades 
de síntese fonêmica não mostrou aumento significativo na análise fonêmica. Além de não haver generalização das habilidades de síntese para as tarefas de análise, esse conhecimento também não garantiu a compreensão do princípio alfabético de escrita.

Estudos apontam que a habilidade de reconhecimento da identidade de fonemas pode ser mais útil que a habilidade de análise e síntese fonêmica (segmentação fonêmica), na aquisição do princípio alfabético. Pré-escolares, que foram ensinados a reconhecer a identidade de fonemas, generalizaram esse conhecimento para outros fonemas além daqueles já ensinados, o que parece demonstrar que se trata de um construto estável e que seu ensino não precisa cobrir todos os fonemas da fala (BYRNE; FIELDING-BARNSLEY, 1990, 1991).

A presente pesquisa teve como objetivo verificar os efeitos da aplicação de um programa de intervenção em habilidades metafonológicas, em ou com crianças cuja língua materna é o português do Brasil e que estão na última série da educação infantil - quando dão início à aprendizagem da linguagem escrita.

Pretende-se responder às seguintes questões: (1) o programa aplicado foi eficaz para o desenvolvimento de habilidades metafonológicas? (2) o desenvolvimento de habilidades metafonológicas favoreceu a aprendizagem da escrita? e (3) a familiaridade do aplicador com as crianças interferiu nos efeitos do programa de intervenção?

\section{Método}

A pesquisa foi realizada em três pré-escolas da rede municipal de educação de um município do estado de São Paulo.

\section{Participantes}

Participaram 90 crianças de 5 classes do último ano da pré-escola, com idades variando entre 5 anos e 4 meses e 6 anos e 5 meses. As classes foram escolhidas de modo a permitir monitorar o efeito-professora na composição dos grupos experimental e controle.

O programa foi aplicado na sala de aula a todas as crianças. No entanto, fizeram parte da pesquisa somente as crianças que no pré-teste demonstraram não ter ainda aprendido a ler e escrever. 
Condições em que o Programa foi Aplicado

- Condição 1: Na escola 1, uma classe regida por uma professora, cujos alunos foram distribuídos para formar o grupo experimental (GE) e o grupo controle (GC), sendo o Programa aplicado pela coordenadora pedagógica.

- Condição 2: Na escola 2, duas classes regidas pela mesma professora em períodos diferentes, sendo que uma classe compôs o grupo experimental (GE) e a outra o grupo controle (GC), sendo o Programa aplicado pelo pesquisador.

- Na escola 3, duas classes do mesmo período, regidas por professoras diferentes, sendo que uma classe constituiu o grupo experimental (GE) e outra o grupo controle (GC), sendo o Programa aplicado pela professora da classe.

O Quadro 1 mostra as três condições de aplicação do programa de intervenção, as classes das crianças participantes, as professoras, grupos experimental e controle, Programa aplicado e aplicadores.

QUADRO 1 - CARACTERÍSTICAS DAS CONDIÇÕES DE APLICAÇÃO DO PROGRAMA DE INTERVENÇÃO EM CONSCIÊNCIA FONOLÓGICA

\begin{tabular}{|c|c|c|c|c|c|}
\hline \multirow[b]{2}{*}{ Condição 1} & \multirow{2}{*}{$\begin{array}{c}\text { Escola } 1 \\
1 \text { classe } \\
\mathrm{N}=25\end{array}$} & Professora & Grupos & Programa & Aplicador \\
\hline & & uma Professora & $\begin{aligned} \mathrm{GE} n & =10 \\
\mathrm{GC} n & =10\end{aligned}$ & $\begin{array}{c}\text { Intervenção } \\
\text { Placebo }\end{array}$ & $\begin{array}{l}\text { Coordenadora } \\
\text { Pesquisador }\end{array}$ \\
\hline Condição 2 & $\begin{array}{c}\text { Escola } 2 \\
2 \text { classes } \\
\text { Classe 1 } \\
\mathrm{N}=27 \\
\text { Classe 2 } \\
\mathrm{N}=25\end{array}$ & $\begin{array}{c}\text { a mesma } \\
\text { professora } \\
\text { nas duas } \\
\text { classes }\end{array}$ & $\begin{array}{l}\text { GE } n=18 \\
\text { GC } n=18\end{array}$ & $\begin{array}{c}\text { Intervenção } \\
\text { Placebo }\end{array}$ & $\begin{array}{l}\text { Pesquisador } \\
\text { Pesquisador }\end{array}$ \\
\hline Condição 3 & $\begin{array}{c}\text { Escola } 3 \\
2 \text { classes } \\
\text { Classe } 1 \\
\mathrm{~N}=26 \\
\text { Classe } 2 \\
\mathrm{~N}=25\end{array}$ & $\begin{array}{l}\text { duas diferentes } \\
\text { professoras, } \\
\text { uma em cada } \\
\text { classe }\end{array}$ & $\begin{aligned} \mathrm{GE} n & =17 \\
\mathrm{GC} n & =17\end{aligned}$ & $\begin{array}{c}\text { Intervenção } \\
\text { Placebo }\end{array}$ & $\begin{array}{l}\text { Professora da } \\
\text { classe } \\
\text { Professora da } \\
\text { classe }\end{array}$ \\
\hline
\end{tabular}




\section{Delineamento e Procedimentos}

A pesquisa constou de um delineamento quase experimental em três fases: pré-teste, intervenção e pós-teste.

As provas de avaliação do pré-teste foram aplicadas no início do ano escolar, na segunda quinzena do mês de fevereiro, a todas as crianças da sala, pela própria pesquisadora, em sessões individuais, com duração de aproximadamente 30 minutos. O Programa de intervenção foi aplicado na semana seguinte ao pré-teste e teve duração de 12 semanas e um total de 32 sessões. O pós-teste foi feito na semana seguinte ao encerramento do Programa de intervenção.

\section{a- Pré-teste:}

Constou de avaliação da leitura, da escrita e de habilidades metafonológicas. A tarefa de leitura teve a finalidade de selecionar os participantes, uma vez que só permaneceriam como sujeitos do estudo as crianças que mostrassem não ter ainda conhecimento do sistema alfabético de leitura. A tarefa de escrita teve duas finalidades: selecionar os participantes (participaram apenas as crianças que não dominavam o princípio alfabético de escrita) e avaliar o desempenho inicial de escrita.

- Avaliação da leitura: A tarefa de leitura foi adaptada de Maluf e Barrera (1997). Constou de quatro cartões com palavras ou enunciados acompanhados de gravuras que, embora se referissem ao que deveria ser lido, não permitiam adivinhação. As palavras e enunciados foram: (1) Natal; (2) Que delícia de sorvete; (3) Chuva; (4) O menino toma sol. A aplicação foi individual.

- Avaliação da escrita: A tarefa de escrita foi a mesma utilizada por Maluf e Barrera (1997). Constou da escrita, sob ditado, das palavras (1) Galo, (2) Pintinho, (3) Onça; (4) Mosquito. A aplicação foi individual.

- Avaliação de habilidades metafonológicas: foi feita utilizando um instrumento composto de 9 tarefas que permitiam avaliar habilidades de (1) identificação da extensão fonológica de palavras; (2) identificação de rimas; (3) identificação de aliterações; (4) consciência de palavras; (5) segmentação silábica; e (6) segmentação fonêmica. As duas primeiras tarefas fazem parte de um instrumento elaborado por Barrera (1995), e as demais elaboradas por nós. A aplicação foi individual.

b- Intervenção em consciência fonológica

O programa de intervenção foi aplicado coletivamente a todos os alunos pertencentes às classes selecionadas, para manter as condições próximas ao cotidiano da sala de aula e evitar a separação entre as crianças. Entretanto, só 
participaram do estudo, no sentido de serem incluídas no pós-teste, as crianças previamente selecionadas por terem demonstrado não dominar o sistema alfabético de leitura e escrita.

O programa consistiu em 32 sessões divididas em cinco unidades de atividades dirigidas à aquisição de habilidades metafonológicas. As atividades foram desenvolvidas com a utilização de músicas, brincadeiras e jogos.

Unidade 1: Atividades de identificação e produção de aliteração (seis sessões).

Unidade 2: Atividades de identificação e produção de rimas (sete sessões).

Para o desenvolvimento dessas duas unidades foram utilizadas músicas que enfatizavam as aliterações e rimas; jogos compostos de cartões com figuras que possibilitavam a formação de pares de palavras que compartilhavam o som inicial ou final e brincadeiras em que as crianças deveriam dizer palavras que tivessem o mesmo som (inicial ou final) de uma palavra alvo.

Unidade 3: Atividades de segmentação de frases em palavras (quatro sessões). Foram utilizados músicas, jogos e brincadeiras com cartões que substituíam palavras de uma frase.

Unidade 4: Atividades de segmentação, omissão e troca silábica (cinco sessões). Foram utilizadas fichas com figuras cujos nomes deveriam ser modificados, por omissão ou troca de sílaba inicial ou final, histórias em que os personagens cometiam omissão ou troca de sílaba das palavras. Unidade 5: Atividades de identificação, troca e omissão de fonemas (dez sessões). Foram realizados jogos e brincadeiras com cartões coloridos e tampinhas que representavam os fonemas a serem omitidos ou trocados.

\section{Programa Placebo}

A fim de aumentar a probabilidade de que os resultados do estudo pudessem ser atribuídos a características do Programa e não simplesmente ao seu caráter de novidade (TROIA, 1999), foram desenvolvidas com as crianças dos grupos controle um conjunto de atividades especialmente elaboradas para esse fim. $\mathrm{O}$ Programa constou de atividades de colagem, desenho e pintura, selecionadas e desenvolvidas de modo a não concorrerem para o desenvolvimento de habilidades metafonológicas.

c- Pós-teste:

Avaliação de habilidades metafonológicas e da escrita. Foram utilizadas as mesmas provas do pré-teste, aplicadas individualmente, na primeira semana após o término da intervenção. Embora fossem considerados apenas os resultados das crianças participantes do estudo, todas as crianças das 
classes envolvidas no estudo foram avaliadas. Essa estratégia foi adotada para atender às expectativas das crianças, que desejavam passar pelas mesmas experiências.

Para análise dos resultados foram atribuídos pontos: zero a quatro pontos para cada uma das quatro palavras escritas; zero a quatro pontos para cada uma das nove questões da tarefa de habilidades metafonológicas. Na análise estatística foi utilizado o teste t de Student.

\section{Resultados}

Para testar a hipótese da semelhança entre os grupos experimental e controle, no que diz respeito às habilidades metafonológicas e de escrita, foi aplicado um modelo geral de análise de variância considerando uma variável dependente e dois fatores. Os resultados mostraram não haver diferenças estatisticamente significativas entre eles, com intervalo de confiança de 0,05 .

A Tabela 1 mostra as médias e os desvios-padrão dos escores nas tarefas de habilidades metafonológicas (HM) e de escrita (TE) das crianças dos grupos experimental (GE) e controle (GC), no pré-teste e no pós-teste, nas condições 1,2 e 3 .

TABELA 1 - MÉDIAE DESVIO-PADRÃONAS TAREFAS DE HABILIDADES METAFONOLÓGICAS (THM) E NAS TAREFAS DE ESCRITA (TE) DAS CRIANÇAS DOS GRUPOS EXPERIMENTAL (GE) E CONTROLE (GC) NO PRÉ-TESTE E NO PÓS-TESTE NAS CONDIÇÕES 1, 2 E 3

\begin{tabular}{c|c|c|c|c|c|c|c|c|c|c}
\hline \multicolumn{9}{c|}{ Pré-Teste } & \multicolumn{4}{c}{ Pós-Teste } \\
\hline \multicolumn{2}{c|}{} & \multicolumn{2}{c|}{ THM } & \multicolumn{2}{c|}{ TE } & \multicolumn{2}{c}{ THM } & \multicolumn{2}{c}{ TE } \\
\hline Condição & Grupo & N & Média & dp & Média & dp & Média & dp & Média & dp \\
\hline \multirow{3}{*}{1} & GE & 10 & 6,9 & 5,2 & 3,7 & 2,1 & 21,1 & 7,1 & 6,3 & 1,7 \\
& GC & 10 & 4,5 & 2,9 & 3,4 & 2,1 & 12,0 & 6,2 & 4,6 & 1,0 \\
\hline \multirow{3}{*}{2} & GE & 18 & 4,2 & 2,2 & 3,1 & 1,5 & 19,2 & 6,0 & 6,1 & 1,8 \\
& GC & 18 & 5,1 & 2,6 & 4,4 & 1,1 & 11,3 & 6,3 & 5,2 & 1,6 \\
\hline \multirow{3}{*}{3} & GE & 17 & 4,8 & 2,5 & 3,5 & 1,4 & 17,8 & 5,4 & 6,1 & 1,4 \\
& GC & 17 & 4,6 & 1,9 & 3,6 & 1,1 & 12,4 & 5,3 & 4,8 & 1,2 \\
\hline
\end{tabular}


Eficácia do Programa para o Desenvolvimento de Habilidades Metafonológicas

A comparação entre os resultados obtidos pelas crianças antes e depois da intervenção, utilizando-se o teste t de Student, mostrou avanços significativos, nas três condições em que o Programa foi aplicado (Condição 1: $t=-5,09$ e p=0,00; Condição 2: $t=-9,96$ e $p=0,00$; Condição $3: t=-8,90$ e $p=0,00)$, o que permitiu afirmar que o procedimento utilizado atingiu o objetivo de produzir avanços nas habilidades metafonológicas.

Os resultados obtidos no pós-teste mostraram que as crianças que passaram pela intervenção obtiveram maiores avanços nas habilidades metafonológicas do que as do grupo controle, nas três condições de aplicação do Programa $(\mathrm{C} 1$ : $\mathrm{t}=3,03$ e $\mathrm{p}=0,01 ; \mathrm{C} 2: \mathrm{t}=3,84$ e $\mathrm{p}=0,00 ; \mathrm{C} 3: \mathrm{t}=2,93$ e $\mathrm{p}=0,01)$.

Essas conclusões permitem afirmar que as crianças se beneficiaram do Programa de intervenção nas três condições em que foi aplicado, tornandose mais hábeis em refletir e manipular unidades sonoras da fala. Portanto, o Programa mostrou-se eficaz em promover um melhor desempenho nas habilidades de julgamento da extensão fonológica de palavras, identificação e produção de rimas e aliterações, segmentação de sentenças em palavras, segmentação de palavras em sílabas e omissão de consoante inicial.

\section{Eficácia do Programa para o Favorecimento de Habilidades de Escrita}

O teste $t$, aplicado à diferença das médias na prova de escrita do pré e pós-teste dos grupos experimentais, mostrou que no pós-teste as crianças apresentaram melhor desempenho na escrita das palavras ditadas, nas condições 1 , 2 e $3(\mathrm{C} 1: \mathrm{t}=-3,03$ e $\mathrm{p}=0,01 ; \mathrm{C} 2: \mathrm{t}=-5,31$ e $\mathrm{p}=0,00$ e $\mathrm{C} 3: \mathrm{t}=-5,2$ e $\mathrm{p}=0,00)$ de aplicação do Programa.

Quando comparados os pós-testes dos grupos experimental e controle, verificou-se que, nas condições 1 e 3 , as crianças que passaram pela intervenção estavam escrevendo melhor do que as que não passaram por essa experiência $(\mathrm{C} 1: \mathrm{t}=2,75$ e $\mathrm{p}=0,01 ; \mathrm{C} 3: \mathrm{t}=2,93$ e $\mathrm{p}=0,01)$.

$\mathrm{Na}$ condição 2, a diferença na escrita das crianças após a intervenção não foi estatisticamente significativa. Para tentar explicar esse resultado divergente, procedeu-se à aplicação de um teste de igualdade de variâncias e diferença de médias. Nesse caso, a diferença das médias mostrou que havia, no pré-teste, um desempenho melhor do grupo controle na prova de escrita $(\mathrm{t}$ $[34]=-2,84 ; p=0,01)$. Se levarmos em consideração essa diferença inicial, a interpretação dos resultados permite sustentar a hipótese de um provável 
avanço maior das crianças do grupo experimental em relação às do grupo controle, na prova de escrita.

\section{Interferência da Familiaridade do Aplicador na Eficácia do Programa}

Parte importante das condições de aplicação do Programa é o aplicador do mesmo: a coordenadora pedagógica, o pesquisador e a professora da classe. Para analisar o possível efeito desse fator, foram comparados os pós-testes das crianças dos três grupos experimentais nas provas de habilidades fonológicas e de escrita.

TABELA 2 - MÉDIAS E DESVIOS-PADRÃO DAS PROVAS DE CONSCIÊNCIA FONOLÓGICA (CF) E DE ESCRITA(PE) DAS CRIANÇAS DO GRUPO EXPERIMENTAL (GE) NO PÓS-TESTE 1 EM CADA UMA DAS CONDIÇÕES DE APLICAÇÃO DO PROGRAMA (C1, C2 E C3)

\begin{tabular}{c|c|c|c|c}
\hline Prova & Condição & $\mathbf{N}$ & Média & Desvio-padrão \\
\hline $\mathrm{CF}$ & 1 & 10 & 21,1 & 7,1 \\
\hline & 2 & 18 & 19,2 & 6 \\
\hline & 3 & 17 & 17,8 & 5,4 \\
\hline $\mathrm{PE}$ & 1 & 10 & 6,3 & 1,7 \\
\hline & 2 & 18 & 6,1 & 1,8 \\
\hline & 3 & 17 & 6,1 & 1,4 \\
\hline
\end{tabular}

Em consciência fonológica, o teste-t para igualdade das médias dos grupos experimentais nas condições 1 e 2 , nas condições 1 e 3 e nas condições 2 e 3 , indicou uma diferença não significativa entre essas condições de aplicação do Programa $(\mathrm{t}=0,74$ e $\mathrm{p}=0,47 ; \mathrm{t}=1,37$ e $\mathrm{p}=0,18 ; \mathrm{t}=0,75$ e $\mathrm{p}=0,46)$. Dessa forma, conclui-se que a familiaridade do aplicador não foi fator determinante do bom resultado encontrado em consciência fonológica após a aplicação do Programa.

$\mathrm{Na}$ tarefa de escrita, o grau de familiaridade do aplicador também não apareceu como fator relevante. $O$ teste $t$ para igualdade das médias nas condições 1 e 2 , nas condições 1 e 3 e nas condições 2 e 3 revelou uma diferença não significativa na tarefa de escrita das crianças $(\mathrm{t}=0,27$ e $\mathrm{p}=0,79 ; \mathrm{t}=0,39$ e $\mathrm{p}=0,70$; $\mathrm{t}=0,09$ e $\mathrm{p}=0,79$ ).

Assim, conclui-se que os ganhos observados nas habilidades metafonológicas e na escrita se devem às características do Programa e não à familiari- 
dade do aplicador, isto é, os resultados não parecem variar em função do fato da aplicação ser feita pela própria professora ou por outros profissionais capacitados, embora com menor grau de familiaridade com as crianças.

Esses resultados também sugerem que o tamanho do grupo não constituiu um fator relevante na aplicação do Programa. Tanto o grupo considerado pequeno (condição 1 com 10 participantes) como os grupos maiores (condições 2 e 3 em que o Programa foi aplicado a todas as crianças da classe) beneficiaram-se do Programa.

\section{Considerações finais}

Os resultados confirmaram análises de investigações anteriores, mostrando que as habilidades metafonológicas têm um importante papel facilitador no início da aquisição da linguagem escrita e podem ser desenvolvidas com o uso de programas de intervenção (LUNDBERG; FROST; PETERSEN, 1988; SCHNEIDER; KÜSPERT; ROTH; VISE; MARX, 1997; HOHN; EHRI, 1983).

Nas três condições em que o Programa de intervenção foi aplicado, observou-se o favorecimento das habilidades metafonológicas nas crianças, bem como melhores resultados na aprendizagem da linguagem escrita. Os ganhos observados ocorreram com diferentes aplicadores, ou seja, quando a aplicação foi feita pela professora ou por outros profissionais capacitados, embora com menor grau de familiaridade com as crianças. Observou-se também que o Programa foi eficiente tanto quando aplicado ao grupo de 10 quanto ao grupo de 27 crianças.

As análises revelam, como já evidenciado em outras pesquisas da área, que o reconhecimento por parte da criança, de que a fala é segmentada em palavras e que estas podem ser segmentadas em unidades menores, é um elemento facilitador da alfabetização (ANTHONY; LONIGAN; DRISCOLL; PHILLIPS; BURGESS, 2003). Para aprender a ler e escrever, é importante que ela compreenda que é possível usar marcas para registrar significados e que essas marcas podem ser lidas; que os sons da fala podem ser representados através de letras e que essas letras e conjuntos de letras podem ser reproduzidos oralmente.

Do ponto de vista pedagógico, esta pesquisa mostrou que o conhecimento do sistema alfabético de escrita pode ser facilitado através de atividades lúdicas, agradáveis e interessantes, que envolvam a identificação e produção de rimas e aliterações, segmentação lexical, bem como atividades de segmentação, omissão 
e troca de sílabas e fonemas. Embora a pesquisa demonstre o papel facilitador dessas práticas, isto não significa que, na sua ausência, as crianças não possam aprender a ler e escrever, mas sim que a presença de tais práticas, mesmo antes do ensino formal, facilita o processo de aprendizagem.

É importante que os professores de educação infantil e de alfabetização reconheçam a importância das habilidades metafonológicas para o processo de aquisição da linguagem escrita e, consequentemente, desenvolvam práticas de ensino que incorporem atividades de linguagem oral, como trocadilhos, músicas e poesias rimadas e jogos de linguagem, a fim de que a criança compreenda o caráter segmental da linguagem e a relação entre letra e som.

\section{REFERÊNCIAS}

ANTHONY, J. L.; LONIGAN, C. J.; DRISCOLL, K.; PHILlIPS, B. M.; BURGESS, S. R. Phonological sensivity: A quasi-parallel progression of word structure units and cognitive operations. Reading Research Quarterly, v. 38, n. 4, p. 470-487, 2003.

BALL, E.; BLACHMAN, B. A. Does phoneme awareness training in kindergarten make a difference in early word recognition and developmental spelling? Reading Research Quarterly, 24, p. 49-66, 1991.

BRADLEY, L.; BRYANT, P. Categorizing sounds and learning to read a casual connection. Nature, 301, p. 419-421, 1983.

BYRNE, B.; FIELDING-BARNSLEY, R. Acquiring the alphabetic principle: A case for teaching recognition of phoneme identity. Journal of Educational Psychology, v. 82, p. 805-812, 1990.

. Evaluation of a program to teach phonemic awareness to young children. Journal of Educational Psychology, v. 83, p. 451-455, 1991.

CAPELLINI, S. A.; CIASCA, S. M. Eficácia do programa de treinamento com a consciência fonológica em crianças com distúrbios de leitura e escrita e distúrbio de aprendizagem. Temas sobre Desenvolvimento, 52, p. 4-10, 2000.

CAPOVILlA, A. G. S.; CAPOVILlA, F. C. Problemas de leitura e Escrita. São Paulo: Memnon, 2000. 
CARDOSO-MARTINS, C. Sensitivity to rhimes, syllables, and phonemes in literacy acquisiton in Portuguese. Reading Research Quarterly, v. 30, n. 4, p. 808-828, 1995.

CARNIO, M. S.; SANTOS, D. Evolução da consciência fonológica em alunos de ensino fundamental. Pró-Fono Revista de Atualização Científica, v. 17, n. 2, p. 195-200, 2005.

CUNNINGHAM, A. E. Explicit versus implicit instrution in phonemic awareness. Journal of Experimental Child Psychology, v. 50, p. 429-444, 1990.

HATCHER, P. J.; HULME, C. Phoneme, rhymes and intelligence as predictors of children's responsiveness to remedial reading instruction: evidence from a longitudinal intervention study. Journal of Experimental Child Psychology, v. 72, p. 130-153, 1999.

HESTER, E.; HODSON, B. The role of phonological representation in decoding skills of young readers. Child Language Teaching and Therapy, UK, v. 20, p. 115-133, 2004.

HOHN, W. E.; EHRI, L. C. Do alphabet letters help prereaders acquire phoneme segmentation skill? Journal of Educational Psychology, v. 75, n. 5, p. 752-762, 1983.

JONG, P. F.; VAN DER LEIJ, A. Spacific contribuitions of phonological abilities to early reading acquisition: results from a dtch latent variable longitudinal study. Journal of Educational Psychology, v. 91, n. 3, p. 450-476, 1999.

JUEL, C. Learning to read and write: a longitudinal study of 54 children fron first throug fourth grades. Journal of Educational Psychology, 80, p. 437-447, 1988.

LIBERMAN, I. Y.; SHANKWEILER, D.; FISCHER, F. W.; CARTER, B. Explicit syllable and phoneme segmentation in the yong child. Journal of Experimental Child Psychology, v. 18, p. 201-212, 1974.

LUNDBERG, I.; FROST J.; PETERSEN, O. P. Effects of an extensive program for stimulating phonological awareness in preschool children. Reading Research Quarterly, v. 23, p. 263-268, 1988.

MALUF, M. R.; BARRERA, S. D. Consciência fonológica e linguagem escrita em pré-escolares. Psicologia: Reflexão e Crítica, Porto Alegre, v. 10, n. 1, p. 125-145, 1997. 
MANN, V. A.; LIBERMAN, I. Phonological awareness and verbal short-term memory. Journal of Learning Disability, v. 17, p. 592-599, 1984.

MORAIS, J. A arte de ler. São Paulo: Editora Unesp, 1996.

MORAIS, J.; CARY, L.; ALEGRIA, J.; BERTELSON, P. Does Awareness of speech as a sequence of phones arise spontaneously? Cognition, v. 7, p. 323-331, 1979.

MURRAY, B. A. Gaining alphabetic insight: is phoneme manipulation skill or identity knowledge causal? Journal of Educational Psychology, v. 90, p. 461-475, 1998.

MUTER, V.; HULME, C.; SNOWLING, M. Segmentation, not rhyming, predicts early progress in learning to read. Journal of Experimental Child Psychology, v. 71, p. 3-27, 1998.

NATION, K. \& HULME, C. Phonemic segmentation, not onset-rime segmentation, predicts early reading and spelling skills. Reading Research Quarterly, v. 32, n. 2, p. 154-167, 1997.

PAULA, G. R.; MOTA, H. B.; KESKE-SOARES, M. A terapia em consciência fonológica no processo de alfabetização. Pró-Fono Revista de Atualização Cientifica, v. 17, n. 2, p. 175-184, 2005.

PERFETTI, C. A.; BECK, I.; BELL, L. C.; HUGHES, C. Phonemic knouedge and learning to read are reciprocal: a longitudinal study of first grade children. Merril-Palmer Quarterly, v. 33, p. 283-219, 1987.

SANTOS, M. J. Consciência Fonológica e Educação Infantil: aplicação de um programa de intervenção e seus efeitos na aquisição da escrita. Tese de Doutorado (Psicologia da Educação) - Pontifícia Universidade Católica de São Paulo, 2004.

SCHNEIDER, W.; KÜSPERT, Roth; VISÉ, M.; MARX, H. Short-and long-term effects of training phonological awareness in kindergarten: evidence from two german studies. Journal of Experimental Child Psychology, v. 66, p. 311-340, 1997.

SNOWLING, M. J.; HULME, C.; SMITH, A.; THOMAS, J. The effects of phonetic similarity and list length on children's sound categorization performance. Journal of Experimental Child Psychology, v. 58, p. 160-180, 1994.

STALL, S.; MURRAY, B. Defining phonological awareness and its relationship with early reading. Journal of Educational Psychology, v. 86, p. 221-234, 1994. 
STANOVICH, K. E. Speculations on the causes and consequences of individual differences in early reading acquisition. In: GOUGH, P.; EHRI, L.; TREIMAN, R. (Org.). Reading Acquisition, Hillsdale, NJ: Erlbaum, 1992, p. 65-106.

. The Matthew effects in reading: some consequences for individual differences in the acquisition of literacy. Reading Research Quarterly, v. 21, p. $360-407,1986$.

TORGESEN, J. K.; MORGAN, S. T.; DAVIS, C. Effects of two types of phonological awareness training on word learning in kindergarten children. Journal of Educational Psychology, v. 84, p. 364-370, 1992.

TORGESEN, J. K.; DAVIS, C. Individual difference variables that predict response to training in phonological awareness. Journal of Experimental Child Psychology, v. 63, p. 1-21, 1996.

TORGESEN, J. K.; WAGNER, R. K.; RAShOtTE, C. A.; ROSE, E.; LINDAMOOD, P.; CONWAY, T.; GARVAN, C. Preventing reading failure in young children with phonological processing disabilities: group and individual response to instruction. Journal of Educational Psychology, v. 91, n. 4, p. 579-593, 1999.

TREIMAN, R. The role of intrasyllabic units in learning to read and spell. In: GOUGH, P.; EHRI, L.; TREIMAN, R. (Org.). Reading Acquisition, Hillsdale, NJ: Erlbaum, 1992.

TROIA, G. A. Phonological awareness intervention research: a critical review of the experimental methodology. Reading Research Quarterly, v. 34, n. 1, p. 28-52, 1999.

WAGNER, R. K.; TORGESEN, J. K. The nature phonological processing and its causal role in the acquisition of reading skills. Psychological Bulletin, v. 101, p. 192-212, 1987.

WESSELING, R.; REITSMA, P. Preschool phonological representations and development of reading skills. Annals of Dyslexia, v. 51, p. 203-229, 2001.

Texto recebido em 25 de junho de 2010.

Texto aprovado em 21 de julho de 2010. 